\title{
Distribution of High-risk Human Papillomavirus Genotypes in Cervical Secretions in Punjab
}

\author{
Sadia Minhas ${ }^{1,6}$, Muhammad Kashif ${ }^{2}$, Zobaria Rehman ${ }^{3}$, Muhammad Bilal Pasha ${ }^{4}$, Muhammad Idrees ${ }^{5}$ and \\ Farheen Ansari ${ }^{6}$ \\ ${ }^{1}$ Department of Oral Pathology, Akhther Saeed Medical and Dental College, Lahore, Pakistan \\ ${ }^{2}$ Department of Oral Pathology, Bakhtawar Amin Medical and Dental College, Multan, Pakistan \\ ${ }^{3}$ Centre of Applied Molecular Biology, University of Punjab, Lahore, Pakistan \\ ${ }^{4}$ Department of Pathology, Bakhtawar Amin Medical and Dental College, Multan, Pakistan \\ ${ }^{5}$ Centre of Excellence in Molecular Biology (CEMB), University of Punjab, Lahore, Pakistan \\ ${ }^{6}$ Institute of Molecular Biology \& Biotechnology (IMBB), University of Lahore, Lahore, Pakistan
}

\begin{abstract}
Objective: To determine the high risk-human papillomavirus (HR-HPV) prevalence, genotype distribution, and associated risk factors in women with general gynaecological problems.

Study Design: Descriptive, cross-sectional study.

Place and Duration of Study: Lady Wallingdon Hospital, Lahore from August 2018 to May 2019.

Methodology: One hundred females with general gynaecological problems constituted the sample. Real-time polymerase chain reaction and liquid base cytology were performed for HR-HPV detection, genotyping, and cytological changes in cervical samples.

Results: The overall prevalence of HR-HPV was found to be 57\%, with the most frequent HR-HPV genotypes HPV 16 (18\%), HPV $18(6 \%)$, and HPV 45 (1\%). The total prevalence of single HR-HPV infection was $25 \%$; whereas, multiple co-infection of HR-HPV was seen in $32 \%$ of cases. In total, $81 \%$ of these women had normal cytology and $19 \%$ had cytologic abnormalities. A significant association was observed between HR-HPV infection and general gynaecological complaints and between HR-HPV infection and cytological abnormalities $(p<0.001)$.

Conclusion: The HR-HPV prevalence was 57\% with the most prevalent genotypes were HPV 16 and HPV 18. The results will also aid the identification of HR-HPV 51 and 31 genotypes, which should be added in the multi-type HPV vaccines.
\end{abstract}

Key Words: Cancer of cervix, Human papillomavirus, Papanicolaou test, Pakistan.

How to cite this article: Minhas S, Kashif M, Rehman Z, Pasha MB, Idrees M, Ansari F. Distribution of High-risk Human Papillomavirus Genotypes in Cervical Secretions in Punjab. J Coll Physicians Surg Pak 2021; 31(07):786-791.

\section{INTRODUCTION}

Worldwide, human papillomavirus (HPV) is one of the most widespread sexually transmitted infections; approximately $65-100 \%$ of sexually active people are infected with HPV sooner or later in their lives. ${ }^{1} \mathrm{HPV}$ family consists of more than 200 genotypes, and nearly 13 of them are identified in the female genital tract. ${ }^{1} \mathrm{HPV}$ can be categorised into high-risk $\operatorname{HPV}(16,18,31,33,35,39,45$, $51,52,56,58,59,68,73$, and 82$)$ and low-risk $(6,11,4,40,42,43$, $44,54,61,70,72,81$ and 89 ) types on the basis of their oncogenic potential. ${ }^{1}$

Correspondence to: Dr. Sadia Minhas, Department of Oral Pathology, Akhther Saeed Medical and Dental College,

Lahore, Pakistan

E-mail: sadiawasif81@gmail.com

Received: March 25, 2021; Revised: May 03, 2021; Accepted: May 24, 2021

DOI: https://doi.org/10.29271/jcpsp.2021.07.786
In women with normal cervical epithelium, the prevalence of HPV is about $10.4 \%$ worldwide, and approximately $8 \%$ in Asia. ${ }^{2}$ However, definite prevalence of HPV in particular populations of women is highly related to age and socio-demographic variables. There is significant association between cervical cancer (CC) and HR-HPV. ${ }^{2,3}$ Although most infected women can overcome the virus without presenting any clinical signs and symptoms, permanent HR-HPV infection is the key risk element for progressionto $\mathrm{CC}^{3}$

The incidence of CC in Pakistan has risen from low to moderate risk, and most of these cases are diagnosed at late stages. ${ }^{4}$ According to Abbas et al. (2020), CC was the third most frequent cancer (5.98\%) in Pakistani women. ${ }^{5}$ A recent report by the International Agency for Research on Cancer (IARC) Information Centre on HPV and Cancer stated that, in Pakistan, approximately 5,601 new cases of CC were identified in 2018, with 3,861 deaths. ${ }^{6}$

Although very few studies have been conducted in Pakistan on 
the non-cancer female population to assess the prevalence and incidence of HPV genotypes, studies from Punjab and Karachi have stated a low prevalence of HPV $(15.4 \%, 4.7 \%, 3.26 \%$, $2.8 \%)^{7-10}$ In these women, the most frequent HR-HPV genotypes were HPV-16 and HPV-18..$^{7-9}$

Epidemiological studies are not only important for obtaining a good understanding and evaluation of the risk of CC development in women infected with HR-HPV types, but also for screening and vaccination programmes. In Pakistan, HPV screening is not a common procedure and there are scarce records on HPV prevalence, modes of transmission and risk factors associated with HPV infection. This is mainly due to the social taboos associated with sexually transmitted diseases (STDs) that impose a significant barrier to research. This is the first study of assess the HR-HPV prevalence and genotypes in females with general gynecological problems in the province of Punjab, Pakistan.

The aim of the current study is to explore HR-HPV prevalence, type distribution and risk factors with general gynaecological problems in the local female population in Punjab Province, Pakistan.

\section{METHODOLOGY}

The cross-sectional and descriptive study comprised 100 women with general gynaecological problemsi.e., lowerabdominal pain, post-coital bleeding, vaginal discharge, irregular menstruation and heavy menstrual bleeding (HMB). The patients attended the gynaecological outpatient department (OPD) of Lady Willingdon Hospital, Lahore, from August 2018 to May 2019. All the patients enrolled in this study were informed about the research objectives, expressed their willingness to undergo a per speculum examination for HPV testing and Pap smear preparation, and signed a written informed consent in the local language (Urdu). The protocol of the study and informed consent was approved by the Ethical Review Committee of the University of Lahore (UOL).

Women were included, if they were married and sexually active, without history of hormone therapy in the past six months, not currently menstruating, pregnancy or lactating. Women with a previous history of chemotherapy or radiotherapy for cervical, vulvar or vaginal cancer, immunocompromised, history of cervical or total uterus resection and unmarried women were excluded.

The information on socio-demographic characteristics, i.e., age, education, socio-economic and marital status, potential HPV risk factors like age, age at first intercourse and life time sexual partners were obtained by interviewing the participants on a pre-designed questionnaire (annexure I). Clinical examination and specimen collection were performed according to standardised operating procedures. Cervicovaginal swabs were collected from all participants with a cervical swab collection kit, i.e., Puritan Uni Tranz- $\mathrm{RT}^{\mathrm{TM}}$; and were placed in a 5-ml vial containing $1 \mathrm{ml}$ of universal viral transport medium labelled with the subject's information. Samples were transported to the
Institute of Molecular Biology and Biotechnology (IMBB) Centre for Research in Molecular Medicine (CRiMM) at UOL and stored at $-20^{\circ} \mathrm{C}$ until further processing.

For liquid base cytology ( $L B C$ ), the samples were obtained by using a specialised cytobrush, i.e., a cervix broom brush. The exfoliated cervical cells of the participants were collected by the gynaecologist from the squamo-columnar junction and then rinsed immediately into the container containing ethanol based preservative fluid, provided by the manufacturer (Hunan Lituo).

Viral genomic DNA isolation (if any) from each sample was initiated by using the VIVANTIS GF-1 viral nucleic acid extraction kit as per the manufacturer's instructions and was performed at CRiMM, UOL. The samples were suspended in $50 \mu$ of elution buffer and stored at $-20^{\circ} \mathrm{C}$. The quantity of DNA was assessed on Pico drop (Thomas Scientific), a value of A260/280 and $A 260 / 230$ was considered satisfactory, if it was in the range of 1.8-2.0 and 1.8-2.2, respectively. ${ }^{11}$ Whereas, the quality of DNA was analysed by electrophoresis on a $2 \%$ agarose gel.

A total of 100 samples were tested by 14 Real-TM Quant kit (SACACE Biotechnologies), which is able to identified $14 \mathrm{HR}$ HPV types $(16,18,31,33,35,39,45,51,52,56,58,59,66,68)$. The kit amplified the E6 and E7 regions of HR-HPV types. The kit contained the internal control (human beta-globin gene) to check for the existence of cellular material in the samples, negative control, standards and PCR mix. The temperature profile was created on a Sacace thermo-cycler (Sa'cycler-96) according to themanufacturer's instructions. The RT-PCR procedure was carried out at the Genome Laboratory.

The LBC was processed at Akhter Saeed Medical and Dental College, Lahore, according to the manufacturer's recommendations (Hunan Lituo Biotechnology). The slides were examined by two certified histopathologist. The reporting of the Pap test was done according to the third edition of The Bethesda System forReporting Cervical Cytology. ${ }^{12}$

The statistical analysis was completed using SPSS Statistics version 22 (IBM Corp., Armonk, NY, USA) for the socio-demographic and clinical characteristics. The overall and type-specific prevalence of HR-HPV was calculated. The HR-HPV types from single and multiple infections were calculated independently. The qualitative data were given as frequencies and percentages, Chi-square and Fisher exact tests were used for determining the association between HR-HPV and above-stated characteristics. $\mathrm{P}<0.05$ was considered statistically significant.

\section{RESULTS}

During the period from August 2018 to May 2019, 125 women who visited for routine gynaecological examination were analysed for HR-HPV infection. Out of these, 25 women were excluded because of incomplete data and finally a total of 100 women were enrolled in the study with detailed demographic characteristics given in Table I.

Among the 100 women, 57 were positive for HR-HPV DNA. The 
HR-HPV genotyping showed that $n=25$ (25\%) of women were positive for single HR-HPV types with the most frequent genotype was HPV 16 (18\%); whereas, $n=32$ (32\%) of women were positive for co-infection of HR-HPV types. Dual HPV infection was seen in $n=20(20 \%)$ of women with the most frequent genotypes HPV 16 and 18 (19\%), while $10 \%$ of women had triple HPV infection with HPV 16,18 and 51 and $2 \%$ of women had HR-HPV infection with four types i.e., 16, 18, 51 and 35 . In the present study, the most frequent genotype was HPV 16 followed by HPV 18 , which was noted both in singleinfection as well as in co-infections.

LBC results revealed that $81 \%$ were negative for intraepithelial lesions or malignancy (NILM); whereas, 19\% were presented with cytologic abnormalities i.e., 9\% ASCUS and 10\% low-grade squamous intraepithelial lesion (LSIL). Overall HR-HPV was positive in $46.9 \%$ of the women without cytological abnormalities and this count was $100 \%$ in women with cytological abnormalities (Figurel).

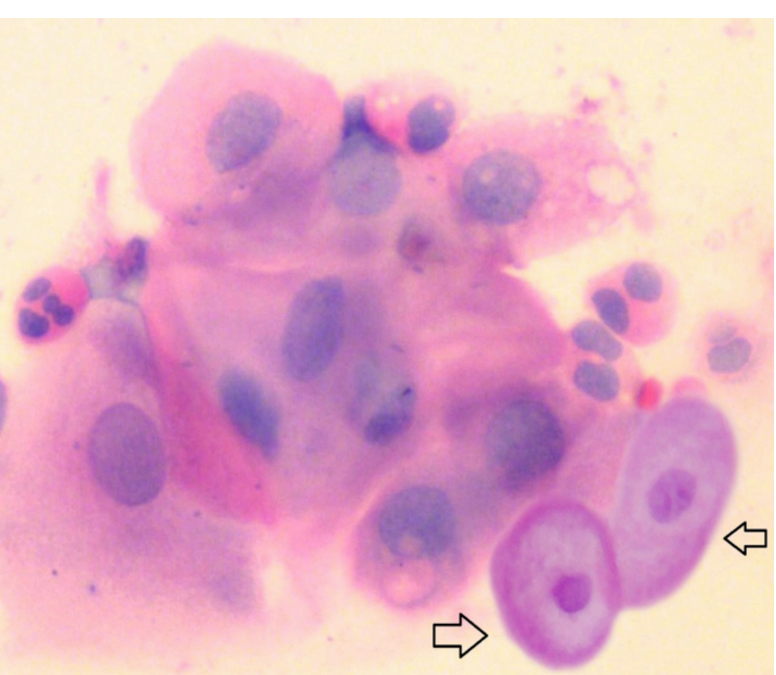

Figure 1: Sheet of squamous cell with nuclear enlargement, mild hyperchromasia and fine chromatin. The koilocytes with perinuclear halo, cytoplasmic thickening, prominent nucleoli, enhanced rim and minimal nuclear enlargement (arrow) are suggestive of ASCUS in Pap smear obtained from cervix ( LBC, 40X).

The most frequent HR-HPV types in 81 cytological normal smears were HPV 16 (18.5\%), whereas in ASCUS the most frequent genotype was HPV 16 (33.3\%) and both HPV 16 and 18 (33.3\%). Moreover, all HR-HPV co-infection was most frequently seen in LSIL with HPV 16 and 18 (40\%) as the most frequent genotypes (Tablell).

Considering the association among HR-HPV and its associated risk factors, it was found that HR-HPV prevalence was most commonly observed in the age group of 25-34 years ( $n=24$, $42.1 \%$ ) with a statistically insignificant between age and HRHPV infection (Tablel).

Among sexual variables, it was found that majority of women who were positive for HR-HPV infection had one-lifetime sexual partner (94.7\%). Likewise, HR-HPV was most frequently observed in women having first intercourse at 14-18 years of age $(n=37,64.9 \%)$ but the association between lifetime sexual partners and age at first intercourse with the incidence of HR$H P V$ was insignificant $(p=0.632$; and $p=0.626$, respectively).

HR-HPV was most commonly observed in women presented with complaint of lower abdominal pain with vaginal discharge ( $n=30,52.6 \%)$. A statistically significant association was observed between HR-HPV infection and gynaecological complaints $(p<0.001)$.

Table I: Shows the demographic and clinical characteristics of the studied group of patients.

\begin{tabular}{|c|c|c|c|}
\hline Characteristics & $\begin{array}{l}N= \\
100(\%)\end{array}$ & $\begin{array}{l}\text { HPV } \\
\text { Positive } \\
\text { N (\%) }\end{array}$ & $p$-value \\
\hline \multicolumn{4}{|l|}{ Age } \\
\hline $15-24$ & $5(5)$ & $2(3.5)$ & \multirow{6}{*}{0.564} \\
\hline $25-34$ & $44(44)$ & $24(42.1)$ & \\
\hline $35-44$ & $38(38)$ & $22(38.6)$ & \\
\hline $45-54$ & $11(11)$ & $8(14)$ & \\
\hline $55-64$ & $1(1)$ & $0(0)$ & \\
\hline Above 65 & $1(1)$ & $1(1.8)$ & \\
\hline \multicolumn{4}{|l|}{ Marital status } \\
\hline Married & $97(97)$ & $55(96.5)$ & 0.671 \\
\hline Widowed & $2(2)$ & $1(1.8)$ & \\
\hline Second Marriage & $1(1)$ & $1(1.8)$ & \\
\hline \multicolumn{4}{|c|}{ Socio-economic status } \\
\hline Poor & $69(69)$ & $38(66.7)$ & \multirow[b]{2}{*}{0.561} \\
\hline Middle & $31(31)$ & $19(33.3)$ & \\
\hline \multicolumn{4}{|l|}{ Source of income } \\
\hline Business & $3(3)$ & $1(1.8)$ & \multirow{5}{*}{0.913} \\
\hline Labor & $87(87)$ & $51(89.5)$ & \\
\hline Teaching & $4(4)$ & $2(3.5)$ & \\
\hline Engineer & $2(2)$ & $1(1.8)$ & \\
\hline Banker & $4(4)$ & $2(3.5)$ & \\
\hline \multicolumn{4}{|l|}{ Education level } \\
\hline Matric & $11(11)$ & $4(7)$ & \multirow{5}{*}{0.248} \\
\hline Middle & $10(10)$ & $8(14)$ & \\
\hline Intermediate & $12(12)$ & $7(12.3)$ & \\
\hline Bachelor & $1(1)$ & $0(0)$ & \\
\hline Uneducated & $66(66)$ & $38(66.7)$ & \\
\hline \multicolumn{4}{|l|}{ Addictive habits } \\
\hline Smoking & $5(5)$ & $3(5.3)$ & \multirow{6}{*}{0.282} \\
\hline Pan & $3(3)$ & $3(5.3)$ & \\
\hline Gutka & $1(1)$ & $0(0)$ & \\
\hline Naswar & $1(1)$ & $0(0)$ & \\
\hline Chalia & $1(1)$ & $0(0)$ & \\
\hline None & $89(89)$ & $51(89.5)$ & \\
\hline \multicolumn{4}{|c|}{ Presenting gynecological complaints } \\
\hline $\begin{array}{l}\text { Lower abdominal pain } \\
\text { and vaginal discharge }\end{array}$ & 37 (37) & $30(52.6)$ & \multirow{7}{*}{$<0.001$} \\
\hline Vaginal discharge & $16(16)$ & $3(5.3)$ & \\
\hline $\begin{array}{l}\text { Heavy menstrual } \\
\text { bleeding and lower } \\
\text { abdominal pain }\end{array}$ & $17(17)$ & $15(26.3)$ & \\
\hline Lower abdominal pain & $14(14)$ & $2(3.5)$ & \\
\hline $\begin{array}{l}\text { Heavy menstrual } \\
\text { bleeding and vaginal } \\
\text { discharge }\end{array}$ & $13(13)$ & $6(10.5)$ & \\
\hline Irregular periods & $2(2)$ & $0(0)$ & \\
\hline Post coital bleeding & $1(1)$ & $1(1.8)$ & \\
\hline \multicolumn{4}{|c|}{ Age at first intercourse } \\
\hline Before 14 years & $15(15)$ & $7(12.3)$ & \multirow{4}{*}{0.626} \\
\hline From $14-18$ years & $62(62)$ & $37(64.9)$ & \\
\hline From $19-23$ years & $16(16)$ & $8(14)$ & \\
\hline From $24-28$ years & $7(7)$ & $5(8.8)$ & \\
\hline \multicolumn{4}{|c|}{ Life time male sexual partners } \\
\hline 1 & $96(96)$ & $54(94.7)$ & \multirow{2}{*}{0.632} \\
\hline 2 & $4(4)$ & $3(5.3)$ & \\
\hline
\end{tabular}


Table II: HR-HPV type distribution in cases with normal and abnormal cytology.

\begin{tabular}{|c|c|c|c|c|c|c|c|c|}
\hline \multirow[t]{2}{*}{ Cervical cytology } & \multicolumn{7}{|c|}{ Hr-Hpv genotypes } & \multirow[b]{2}{*}{$\begin{array}{c}\text { Negative for HR-HPV } \\
\text { n (\%) }\end{array}$} \\
\hline & $\begin{array}{l}\text { HPV } 16 \\
\text { n (\%) }\end{array}$ & $\begin{array}{c}\text { HPV } 18 \\
\text { n (\%) }\end{array}$ & $\begin{array}{c}\text { HPV } 45 \\
\text { n (\%) }\end{array}$ & $\begin{array}{c}\text { HPV } 16 \& 18 \\
n(\%)\end{array}$ & $\begin{array}{c}\text { HPV } 16 \& 51 \\
n(\%)\end{array}$ & $\begin{array}{c}\text { HPV } 16,18 \& 51 \\
n(\%)\end{array}$ & $\begin{array}{c}\text { HPV 16, 18, } 51 \& \\
35 \\
\text { n (\%) }\end{array}$ & \\
\hline Normal $(n=81)$ & $15(18.5)$ & $5(6.2)$ & $1(1.2)$ & $12(14.8)$ & 0 & $5(6.2)$ & 0 & $43(53.1)$ \\
\hline Ascus $(n=9)$ & $3(33.3)$ & $1(11.1)$ & 0 & $3(33.3)$ & 0 & $2(22.2)$ & 0 & 0 \\
\hline Lsil $(n=10)$ & 0 & 0 & 0 & $4(40)$ & $1(10)$ & $3(30)$ & $2(20)$ & 0 \\
\hline
\end{tabular}

\section{DISCUSSION}

Data on HPV infection, its prevalence and type-specific HRHPV infection in women with and without cervical abnormalities is crucial in planning and conducting an early HPV screening and vaccination programme to reduce its disease burden and associated fatal diseases in the country. Pakistan is the $6^{\text {th }}$ most populous country and adds considerably to the world database of CC due to inadequate screening programs and targeted vaccination for HPV infection. ${ }^{13}$

Contrary to previously conducted studies in Pakistan, which reported a low prevalence of HPV among women with normal cytology and general gynaecological problems ranging from $2.17-15.4 \%$, the present study reported the highest prevalence of HR-HPV infection (57\%) among the non-cancer female population with minor gynaecological complaints; ${ }^{7-10}$ whereas, other local studies have reported a higher prevalence of HPV in CC patients $(98.3 \%, 94.8 \%$, $88 \%$, and $87.5 \%$, respectively). ${ }^{4,14-16}$ This low prevalence of HPV in the previous local studies is in striking contrast to the rising trend of $\mathrm{CC}$ in the country, where almost 20 women fall victim to CC daily, making it one of the top 10 countries with the highest female mortality rates. ${ }^{7}$ In 2008 , a meta-analysis of 79 studies comprising a total number of 25,368 women with normal cytology, stated that the overall prevalence of HPV in Asia was $14.4 \% .{ }^{17}$ However, studies from neighbourhood countries, India and China, reported a raised prevalence of cervical HR-HPV infection in normal cytological samples $\left(68.6 \%\right.$ and $57.7 \%$, respectively). ${ }^{18,19}$ Likewise, studies from Brazil and Hong Kong reported a high prevalence ( $53.6 \%$ and $41.7 \%$, respectively) of HPV infection in sexually active adolescents. ${ }^{20,21}$

The current study reported the higher prevalence of HR-HPV in Pakistani females in contrast to previous local studies. ${ }^{7-10}$ This may be because as most of the studies were only from the southern region of Pakistan and hospital-based accrual of patients. Moreover, conventional PCR technique was used for the identification of HPV infection as compared to RT-PCR which is more sensitive and reliable diagnostic technique. The high prevalence of HR-HPV in the current study reflects the HPV profile in the local female population, exposure to multiple risk factors for HPV infection, absence of screening and awareness programs, socio-cultural characteristics, and lack of sensitive diagnostic method for HPV infection.
The analysis of genotype distribution in the present study showed that the most frequent HR-HPV genotypes were 16 and 18 (Figures 1 and 2), which is similar to a local study carried out on the general female population and in CC cases. $^{5,8,9,14,15}$ Likewise, the studies carried out in Tehran and India also reported similar findings. ${ }^{18,22,23}$ However, the present study is in contrast with the local and international studies conducted in Pakistan, China and Hong Kong reporting that HPV 45 (12.5\%), HPV 52 (7.8\%) and HPV 66 (8.3\%) as the most frequent HR-HPV genotypes. ${ }^{7,20,21,24}$ It is important to mention that this study has also reported the rare genotypes HPV 51 and HPV 35 for the first time in the Pakistani population, which may be due to the movement of the labor force to and from those specific regions. Therefore, it is significant to evaluate the HR-HPV genotypes circulating in the Pakistani population, to prevent CC and the introduction of both CC screening and HPV vaccination programmes.

According to this study, among females with general gynaecological problems, $32 \%$ were infected with co-infection of predominantly HPV 16 and 18 which is in line with the local $(40.26 \%)$ and international $(31 \% ; 37.94 \%)$ data; ${ }^{15,20,24}$ whereas, in contrast with the studies carried out in Lahore, Pakistan on CC cases $(0.8 \%)$, India $(23.41 \%)$ and Hong Kong (17.6\%). ${ }^{16,21,23}$ Hence, it is safe to conclude that HPV-16 is the most common genotype in Pakistan as a mono and co-infection.

So far, very limited studies have been published in Pakistan reporting the prevalence of HR-HPV in females with normal cervical cytology. In this study, HR-HPV positivity in women with normal cervical cytological cases was $46.9 \%$ which is in contrast to that reported by Pakistan (2.2\%), Iran (13\%) and Hong Kong (18.1\%). ${ }^{9,21,22}$ Interestingly, it was also observed that among all abnormal cervical cytology $100 \%$ (ASCUS and LSIL) were positive for HR-HPV infection which is comparable with the other published local and international data. ${ }^{9,23,25}$

Considering the age of infected females, the highest HR-HPV infection rate was seen in the age range of 25-34 years (42.1\%) and 35-44 years (38.6\%). However, the frequency of HR-HPV declined from 45 years of age and onwards, which suggests an opposite association between older age and HRHPV incidence. These findings are in accordance with international studies that reported HPV prevalence is more frequent among females at young age and decreases with increasing age..$^{20,24}$ However, the current study data is in contrast to the local and international studies. ${ }^{7,9,23,25}$ The increased HR-HPV prevalence in the younger age $(25-34 \mathrm{y})$ can be possibly 
explained by the fact that the young females are more sexually active displaying an indirect evidence of sexual transmission and decreased sensitivity of the immune system to the HPV infection.

The present study allows for constructing a countrywide map of the HR-HPV type distribution, emphasises the requirement for HPV infection screening and introduction of an HPV vaccination programme. It is also recommended to implement HR-HPV DNA testing routinely in addition to Pap smear during cervical screening.

The data from the current study is too limited to comment more precisely on the distribution and genotypes circulating in the Pakistani female population; but still it provides significant information regarding HR-HPV prevalence and genotypes in the Pakistani population. Hence, there is an urgent need to carry out large scale studies in females with normal cytology to determine the prevalence of HR-HPV genotypes.

\section{CONCLUSION}

The prevalence of HR-HPV (57\%) is higher compared to previously reported studies carried out in the Pakistani female population residing in Lahore; the most frequent genotypes were found to be HR-HPV 16 and 18.

\section{DISCLOSURE:}

This study is a part of thesis of PhD.

\section{PATIENTS' CONSENT:}

The consents of the female participants were taken prior to the sampling.

\section{CONFLICT OF INTEREST:}

The authors declared no conflict of interest.

\section{AUTHORS' CONTRIBUTION:}

SM: Designed the study, sample collected data, performed the procedure, organised and analysed the data, and wrote the final manuscript.

MBP: Carried out analysis.

MK: Analysed the data and proofread the manuscript.

ZR: Performed real time PCR and wrote the segment of manuscript.

MI, FA: Assisted study, reviewed and edited the manuscript.

SM, FA: Assisted in funding arrangement.

All authors finally reviewed and approved the study.

\section{REFERENCES}

1. Farahmand M, Moghoofei M, Dorost A, Abbasi S, Monavari $\mathrm{SH}$, Kiani SJ, et al. Prevalence and genotype distribution of genital human papillomavirus infection in female sex workers in the world: A systematic review and meta-analysis. BMC Public Health 2020; 20(1):1-4. doi: 10.1186/ s12889-020-09570-z

2. De Sanjosé $S$, Diaz M, Castellsagué $X$, Clifford G, Bruni L, Muñoz N, et al. Worldwide prevalence and genotype distribu- tion of cervical human papillomavirus DNA in women with normal cytology: A meta-analysis. Lancet Infectious Diseases 2007; 7(7):453-9. doi: 10.1016/ S14733099(07)70158-5.

3. Boda D, Docea AO, Calina D, Ilie MA, Caruntu C, Zurac S, et al. Human papilloma virus: Apprehending the link with carcinogenesis and unveiling new research avenues. Int J Oncol 2018; 52(3):637-55. doi: 10.3892/ijo.2018.4256.

4. Khan GJ, Naeem HS, Khan S, Jamshaid T, Sajid MI, Bashir I, Jamshaid $M$. Understanding and responsiveness level about cervical cancer and its avoidance among young women of Pakistan. Asian Pac J Cancer Prev 2014; 15(12):4877-83. doi: 10.7314/APJCP.2014.15.12.4877.

5. Abbas G, Shah S, Hanif M, Asghar A, Shafique M, Ashraf K. Cancer prevalence, incidence and mortality rates in Pakistan in 2018. Bull Cancer 2020; 107(4):517-8. doi: 10.1016/j.bulcan.2019.12.011.

6. Bruni L, Albero G, Serrano B, Mena M, Gómez D, Muñoz J, et al. ICO/IARC information centre on HPV and cancer (HPV information centre). Human papillomavirus and related diseases in Pakistan. Summary Rep 2019; 17:2019.

7. Aziz H, Iqbal H, Mahmood H, Fatima S, Faheem M, Sattar $A A$, et al. Human papillomavirus infection in females with normal cervical cytology: Genotyping and phylogenetic analysis among women in Punjab, Pakistan. Int J Infect Dis 2018; 66:83-9. doi: 10.1016/j.ijid.2017.11.009.

8. Abdullah A, Qasim M, Shafiq M, ljaz M, Parveen S, Murtaza $S$, et al. Molecular diagnosis and phylogenetic analysis of human papillomavirus type-16 from suspected patients in Pakistan. Infect Agent Cancer 2016; 11(1):1-7.

9. Raza SA, Franceschi S, Pallardy S, Malik FR, Avan BI, Zafar $A$, et al. Human papillomavirus infection in women with and without cervical cancer in Karachi, Pakistan. Br J Cancer 2010; 102(11):1657-60.

10. Khan S, Jaffer NN, Khan MN, Rai MA, Shafiq M, Ali A, et al. Human papillomavirus subtype 16 is common in Pakistani women with cervical carcinoma. Int J Infect Dis 2007; 11(4):313-7. doi: 10.1016/j.ijid.2006.06.007.

11. Desjardins $P$, Conklin D. Nanodrop microvolume quantitation of nucleic acids. J Vis Exp 2010; 22(45):e2565. doi: $10.3791 / 2565$.

12. Nayar R, Wilbur DC, editors. The Bethesda system for reporting cervical cytology: definitions, criteria, and explanatory notes. Springer 2015.

13. Denny L, Quinn M, Sankaranarayanan R. Chapter 8: Screening for cervical cancer in developing countries. Vaccine 2006; 24 Suppl 3:S71-7. doi: 10.1016/j.vaccine. 2006.05.121.

14. Gul S, Murad S, Javed A. Prevalence of high risk human papillomavirus in cervical dysplasia and cancer samples from twin cities in Pakistan. Int J Infect Dis 2015; 34:14-9. doi: 10.1016/j.ijid.2015.02.018.

15. Siddiqa A, Zainab M, Qadri I, Bhatti MF, Parish JL. Prevalence and genotyping of high risk human papillomavirus in cervical cancer samples from Punjab, Pakistan. Viruses 2014; 6(7):2762-77. doi: 10.3390/ v6072762.

16. Loya A, Serrano B, Rasheed F, Tous S, Hassan M, Clavero $\mathrm{O}$, et al. Human Papillomavirus genotype distribution in 
invasive cervical cancer in Pakistan. Cancers 2016: 8(8):72. doi: 10.3390/cancers8080072.

17. Bao YP, Li N, Smith JS, Qiao YL. Human papillomavirus type distribution in women from Asia: A meta-analysis. Int J Gynecologic Cancer 2008; 18(1):71-9. doi: 10.1111/j. 1525-1438.2007.00959.x.

18. Kapoor CS, Sharma M. Prevalence of HPV infection in reproductive aged female in Delhi NCR region. Clin Epidemiol Glob Health 2020; 8(2):612-5. doi.org/10. 1016/j.cegh. 2019.12.012

19. Vinodhini K, Shanmughapriya S, Das BC, Natarajaseenivasan K. Prevalence and risk factors of HPV infection among women from various provinces of the world. Arch Gynecol Obstet 2012; 285(3):771-7. doi: 10.1007/ s00404-011-2155-8.

20. Wendland EM, Villa LL, Unger ER, Domingues CM, Benzaken AS. Prevalence of HPV infection among sexually active adolescents and young adults in Brazil: The POPBrazil Study. Sci Rep 2020; 10(1):1-0. doi: 10.1038/ s41598-020-61582-2.

21. Chau AM, Chung JK, Ng SW, Tam DC. Prevalence of human papillomavirus infection and its correlation with age and cervical cytology results in Hong Kong. J Hong Kong Instit Med Lab Sci 2017-2018; 15:1-2.

22. Ghaffari SR, Sabokbar T, Mollahajian H, Dastan J, Ramezanzadeh $F$, Ensani $F$, et al. Prevalence of human papillomavirus genotypes in women with normal and abnormal cervical cytology in Iran. Asian Pac J Cancer Prev 2006; 7(4):529-32.

23. Senapati R, Nayak B, Kar SK, Dwibedi B. HPV Genotypes distribution in Indian women with and without cervical carcinoma: Implication for HPV vaccination program in Odisha, Eastern India. BMC Infect Dis 2017; 17(1):1-0. doi: 10.1186/ s12879-016-2136-4.

24. Luo Q, Jiang N, Wu Q, Wang J, Zhong J. Prevalence and genotype distribution of HPV and cervical pathological results in Sichuan Province, China: A three years surveys prior to mass HPV vaccination. Virology J 2020; 17(1):100. doi: 10.1186/s12985-020-01366-2.

25. He L, He J. Distribution of high-risk HPV types among women in Sichuan province, China: A cross-sectional study. $B M C$ Infect Dis 2019; 19(1):1-8. doi: 10.1186/ s12879-019-4038-8. 